S. Goto and Y. Shimoda

Nagoya Math. J.

Vol. 92 (1983), $69-88$

\title{
ON THE GORENSTEINNESS OF REES AND FORM RINGS OF ALMOST COMPLETE INTERSECTIONS
}

\author{
SHIRO GOTO AND YASUHIRO SHIMODA*)
}

\section{§1. Introduction}

Let $A$ be a Noetherian local ring and $p$ a prime ideal in $A$. Let

$$
R(p)=\bigoplus_{i \geq 0} p^{i} \text { and } G(p)=\bigoplus_{i \geq 0} p^{i} / p^{i+1}
$$

and call them, respectively, the Rees ring and the form ring of $p$. The purpose of this paper is to clarify, provided $p$ is an almost complete intersection in $A$ (cf. (2.1) for definition), when the rings $R(p)$ and $G(p)$ are Gorenstein. The main results are contained in the following two theorems (see also Theorem (3.4)):

THEOREM (1.1). The following conditions are equivalent.

(1) $G(p)$ is a Gorenstein ring.

(2) $A$ is a Gorenstein ring and $A / p$ is a Cohen-Macaulay ring.

Theorem (1.2). Let $r=\operatorname{dim} A_{p}$ and assume that $A$ is a CohenMacaulay ring. Then

(1) $R(p)$ is not a Gorenstein ring, if $r \geqq 3$.

(2) Suppose that $r=2$. Then $R(p)$ is a Gorenstein ring if and only if $G(p)$ is a Gorenstein ring.

Similar results for $r \leqq 1$ on the Gorensteinness of $R(p)$ may be found in Section 4 (cf. Lemma (4.1) and Theorem (4.4)).

The proof of Theorem (1.1) (resp. Theorem (1.2)) shall be given in Section 3 (resp. Section 4). We will summarize in Section 2 some basic results on almost complete intersections, which we frequently need to prove the above theorems.

Throughout this paper, let $A$ be a Noetherian local $\operatorname{ring}$ of $\operatorname{dim} A$ $=d$ and $m$ the maximal ideal in $A$. Let $H_{m}^{i}(\cdot)$ denote, for each $i$, the $i^{\text {th }}$ local cohomology functor relative to $m$.

Received June 3, 1982.

*) Both the authors are supported by Grant-in-Aid for Co-operative Research. 


\section{§2. Preliminaries}

First of all, let us recall the definition of almost complete intersections.

Definition (2.1). Let $p$ be a prime ideal of $A$ with $\operatorname{dim} A_{p}=r$. Then $p$ is said to be an almost complete intersection in $A$, if $p$ is minimally generated by $r+1$ elements and if the local ring $A_{p}$ is regular.

For instance, let $d>r \geqq 0$ be integers and let $R$ be a regular local ring of dimension $d+1$. Let

$$
f=a_{1} b+\sum_{i=2}^{r+1} a_{i}^{2} \quad \text { and } P=\left(a_{1}, a_{2}, \cdots, a_{r+1}\right) R,
$$

where $a_{1}, a_{2}, \cdots, a_{d}, b$ is a regular system of parameters for $R$. We put $A=R / f R$ and $p=P / f R$. Then $\operatorname{dim} A_{p}=r$, and the ideal $p$ is an almost complete intersection in $A$. Clearly $A$ is a Gorenstein ring of dimension $d$ and $A / p$ is regular.

ExAMPLE (2.2). Let $k[[X, Y, Z]]$ and $k[[t]]$ be formal power series rings over a field $k$ and let $H$ be a numerical semigroup generated by 3 elements, say $H=\langle a, b, c\rangle$. Let $\varphi: k[[X, Y, Z]] \rightarrow k[[t]]$ be the $k$-algebra map defined by

$$
\varphi(X)=t^{a}, \quad \varphi(Y)=t^{b} \text { and } \varphi(Z)=t^{c} .
$$

We put $p=\operatorname{Ker} \varphi$. Then if the ring $k\left[\left[t^{a}, t^{b}, t^{c}\right]\right]$ is not Gorenstein, the prime ideal $p$ is minimally generated by 3 elements and is an almost complete intersection in $k[[X, Y, Z]]$ (cf. [6]). For instance, let $H=$ $\langle 3,4,5\rangle$. Then

$$
p=\left(X^{3}-Y Z, Y^{2}-X Z, Z^{2}-X^{2} Y\right),
$$

which is a typical example of almost complete intersections in $k[[X, Y, Z]]$.

Let $e(A)$ be the multiplicity of $A$ relative to $m$. Given a finitely generated $A$-module $M$, let $v_{A}(M)$ denote the number of elements in a minimal system of generators for $M$. We put $v(A)=v_{A}(m)$, the embedding dimension of $A$.

Proposition (2.3). Let $A$ be a regular local ring and $p$ a prime ideal of $A$ contained in $m^{2}$. Assume that $A / p$ is a Cohen-Macaulay ring with

$$
v(A / p)=e(A / p)+\operatorname{dim} A / p-1 \text {. }
$$


Then the following conditions are equivalent.

(1) $p$ is an almost complete intersection in $A$.

(2) $e(A / p)=3$.

Proof. By [14, Theorem 1] we get $v_{A}(p)=\left(\begin{array}{l}e \\ 2\end{array}\right)$, where $e=e(A / p)$. Notice that

$$
\begin{aligned}
v_{A}(p)-\operatorname{dim} A_{p} & =v_{A}(p)-(d-\operatorname{dim} A / p) \\
& =v_{A}(p)-(e-1) \\
& =\left(v_{A}(p)-e\right)+1,
\end{aligned}
$$

since $d=e+\operatorname{dim} A / p-1$ by our assumption. Then $p$ is an almost complete intersection in $A$ if and only if $\left(\begin{array}{l}e \\ 2\end{array}\right)=e$, i.e., $e=3$.

Let $H \subset N^{2}$ be a finitely generated additive submonoid of $N^{2}$ with $\operatorname{rank}_{Z} H=2$. Let $k$ be a field and $k[H]$ the monoid algebra of $H$ over $k$. We denote by $t^{h}$, for each $h \in H$, the canonical image of $h$ in $k[H]$. Let $h_{1}, h_{2}, \cdots, h_{v}$ be a minimal system of generators for $H$. Let $R=$ $k\left[X_{1}, X_{2}, \cdots, X_{v}\right]$ be a polynomial ring and let $\varphi: R \rightarrow k[H]$ denote the epimorphism of $k$-algebras defined by $\varphi\left(X_{i}\right)=t^{h_{i}}$ for all $1 \leqq i \leqq v$. We put $A=R_{M}$ and $p=P A$, where

$$
M=\left(X_{1}, X_{2}, \cdots, X_{v}\right) R \quad \text { and } P=\operatorname{Ker} \varphi .
$$

Corollary (2.4). Suppose that the ring $k[H]$ is normal. Then the following conditions are equivalent.

(1) $p$ is an almost complete intersection in $A$.

(2) $v=4$.

Proof. Let $S=k[H]$ and $N=\left(t^{h_{i}} \mid 1 \leqq i \leqq v\right) S$. Clearly $S$ is a CohenMacaulay ring, as it is normal and of dimension 2. Moreover by virtue of [3], we get that $v\left(S_{N}\right)=e\left(S_{N}\right)+1$. Hence as $v=v\left(S_{N}\right)$, the assertion follows from (2.3).

Because the structure of 2-dimensional normal semigroups $H\left(\subset N^{2}\right)$ is completely known (cf., e.g., [1]), one has, by (2.4), numerous examples of almost complete intersections $p$ of height 2 in the regular local ring $A=k\left[X_{1}, X_{2}, X_{3}, X_{4}\right]_{M}$. For instance,

$$
\begin{aligned}
p=\left(X_{3}^{2}-X_{2} X_{4},\right. & \left.X_{1} X_{4}-X_{2}^{2} X_{3}, X_{2}^{3}-X_{1} X_{3}\right) A \\
& \text { where } H=\langle(0,5),(1,2),(3,1),(5,0)\rangle, \\
p=\left(X_{2}^{2}-X_{1} X_{3},\right. & \left.X_{1} X_{4}-X_{2} X_{3}, X_{3}^{2}-X_{2} X_{4}\right) A \\
& \text { where } H=\langle(0,3),(1,2),(2,1),(3,0)\rangle,
\end{aligned}
$$




$$
\begin{aligned}
& p=\left(X_{2}^{3}-X_{1} X_{3}, X_{3}^{2}-X_{2} X_{4}, X_{1} X_{4}-X_{2}^{2} X_{3}^{2}\right) A \\
& \quad \text { where } H=\langle(0,8),(1,3),(3,1),(8,0)\rangle .
\end{aligned}
$$

Now let us fix a prime ideal $p$ of a Cohen-Macaulay local ring $A$ and assume that $p$ is an almost complete intersection. We put $r=\operatorname{dim} A_{p}$.

The next result (2.5) is already known (cf., e.g., [4] and [9]). However we need it so frequently that we shall give a proof for completeness.

Lemma (2.5). There exist elements $a_{1}, a_{2}, \cdots, a_{r+1}$ of $A$ such that

(1) $p=\left(a_{1}, a_{2}, \cdots, a_{r+1}\right)$ and $p A_{p}=\left(a_{1}, a_{2}, \cdots, a_{r}\right) A_{p}$,

(2) $a_{1}, a_{2}, \cdots, a_{r}$ is an A-regular sequence, and

(3) $\left(a_{1}, \cdots, a_{r}\right): a_{r+1}=\left(a_{1}, \cdots, a_{r}\right): a_{r+1}^{2}$.

Proof. Choose elements $a_{1}, a_{2}, \cdots, a_{r+1}$ of $A$ so that $p=\left(a_{1}, a_{2}, \cdots, a_{r+1}\right)$, $p A_{p}=\left(a_{1}, a_{2}, \cdots, a_{r}\right) A_{p}$, and $h t_{A}\left(a_{1}, a_{2}, \cdots, a_{r}\right)=r$ (cf. [12, p. 427, Corollary 2]). Then $a_{1}, a_{2}, \cdots, a_{r}$ is certainly an $A$-regular sequence, as $A$ is CohenMacaulay.

Let us prove the assertion (3). Passing to the ring $A /\left(a_{1}, a_{2}, \cdots, a_{r}\right)$, we may assume that $r=0$. Let $x \in A$ and suppose that $a_{1} x \neq 0$. We choose $P \in \operatorname{Ass} A$ so that $a_{1} x \neq 0$ in $A_{P}$. Then as $a_{1} \neq 0$ in $A_{P}$, we get $P \ngtr p=\left(a_{1}\right)$ (recall that $A_{p}$ is a field). Consequently $a_{1}^{2} x \neq 0$ in $A_{P}$, since $a_{1}$ is a unit and $x \neq 0$ in $A_{P}$. Thus $a_{1}^{2} x \neq 0$ in $A$ and we get that $(0): a_{1}=(0): a_{1}^{2}$ as required.

Let $a_{1}, a_{2}, \cdots, a_{r+1}$ be elements of $A$ obtained by Lemma (2.5). Then by the conditions (2) and (3) we get

$$
\left(a_{1}, \cdots, a_{i-1}\right): a_{j}=\left(a_{1}, \cdots, a_{i-1}\right): a_{i} a_{j},
$$

whenever $1 \leqq i \leqq j \leqq r+1$. Accordingly we immediately have the following

Corollary (2.6) ([9]). The equality

$$
\left(a_{1}, \cdots, a_{i}\right) \cap p^{n}=\left(a_{1}, \cdots, a_{i}\right) p^{n-1}
$$

holds for any integers $1 \leqq i \leqq r+1$ and $n$.

Corollary (2.7) (cf., e.g., [8, p. 488, Remark]). Let $S(p)$ denote the symmetric algebra of the A-module $p$. Then

$$
S(p) \cong R(p)
$$

as A-algebras. 
We put $s=\operatorname{depth} A / p$. Let $N=m R(p)+[R(p)]_{+}$(resp. $M=m G(p)$ $\left.+[G(p)]_{+}\right)$be the unique graded maximal ideal of the Rees ring $R(p)$ (resp. the form ring $G(p)$ ) of $p$. Then as is well-known,

$$
\operatorname{dim}[R(p)]_{N}=d+1 \text { and } \operatorname{dim}[G(p)]_{M}=d
$$

(cf., e.g., [17]). Moreover we have

Proposition (2.8) ([4]). (1) $\operatorname{depth}[G(p)]_{M}=\min \{d, s+r+1\}$.

(2) $\operatorname{depth}[R(p)]_{N}=\min \{d, s+2\} \quad(r=0)$,

$$
=\min \{d+1, s+r+2\} \quad(r>0) .
$$

By virtue of this result we can easily estimate, in terms of $s=\operatorname{depth} A / p$ and $r=\operatorname{dim} A_{p}$, when the rings $R(p)$ and $G(p)$ are Cohen-Macaulay.

We close this section with one more remark. Let $B$ be, for a moment, an arbitrary Noetherian local ring with maximal ideal $n$ and $E$ a Cohen-Macaulay $B$-module of dimension $k$. We put

$$
r_{B}(E)=\operatorname{dim}_{B / n} \operatorname{Ext}_{B}^{k}(B / n, E)
$$

and call it the type of $E$.

Lemma $(2.9)([7,1.22])$. Let $x$ be an E-regular element of $B$. Then $r_{B}(E)=r_{B}(E / x E)$.

We denote $r_{B}(B)$ simply by $r(B)$, in case $B$ itself is Cohen-Macaulay. When $B$ is not necessarily local, we put

$$
r(B)=\sup _{P \in \operatorname{Spec} B} r\left(B_{P}\right)
$$

and call it the global type of $B$ (cf. [2]). Notice that the invariant $r(B)$ measures how the ring $B$ differs from Gorenstein rings: $B$ is a Gorenstein ring if and only if $B$ is Cohen-Macaulay and $r(B)=1$.

\section{$\S 3$. The Gorensteinness of form rings $G(p)$ and Proof of Theorem (1.1)}

Similarly as in the previous section, we suppose that $A$ is a CohenMacaulay local ring. Let $p$ be a prime ideal in $A$ and assume that $p$ is an almost complete intersection. We put

$$
\begin{aligned}
& R=R(p), \text { the Rees ring of } p, \\
& G=G(p), \text { the form ring of } p, \\
& N=m R+R_{+}, \text {the unique graded maximal ideal of } R,
\end{aligned}
$$




$$
\begin{aligned}
M & =m G+G_{+}, \text {the unique graded maximal ideal of } G, \\
r & =\operatorname{dim} A_{p}, \\
s & =\operatorname{depth} A / p .
\end{aligned}
$$

Usually we identify $R$ with the $A$-subalgebra

$$
A[a T \mid a \in p]
$$

of $A[T]$, where $T$ is an indeterminate over $A$. Let $a_{1}, a_{2}, \cdots, a_{r-1}$ be elements of $A$ obtained by Lemma (2.5). We put

$$
I=\left(a_{1}, \cdots, a_{r}\right): a_{r+1} \text {. }
$$

To begin with, we need the following

Proposition (3.1). $\operatorname{dim} A / I=d-r$ and

$$
\operatorname{depth} A / I=\min \{s+1, d-r\} \text {. }
$$

Proof. Since $I=\left(a_{1}, \cdots, a_{r}\right): a_{r+1}$ and $p=\left(a_{1}, \cdots, a_{r}, a_{r+1}\right)$, we get a short exact sequence

$$
0 \longrightarrow A / I \longrightarrow A /\left(a_{1}, a_{2}, \cdots, a_{r}\right) \longrightarrow A / p \longrightarrow 0
$$

of $A$-modules. As $s=\operatorname{depth} A / p$ and as $A /\left(a_{1}, a_{2}, \cdots, a_{r}\right)$ is a CohenMacaulay ring of dimension $d-r$, the second assertion follows from the sequence (\#).

For the first assertion, notice that the ideal $I$ consists of zerodivisors in $A /\left(a_{1}, a_{2}, \cdots, a_{r}\right)$. Therefore we may choose $P \in \mathrm{Ass}_{A} A /\left(a_{1}, a_{2}\right.$, $\left.\cdots, a_{r}\right)$ so that $P \supset I$, whence $\operatorname{dim} A / I \geqq \operatorname{dim} A / P=d-r$. As $\operatorname{dim} A / I$ $\leqq \operatorname{dim} A /\left(a_{1}, a_{2}, \cdots, a_{r}\right)=d-r$, the required equality $\operatorname{dim} A / I=d-r$ now follows.

Corollary (3.2). Assume that $r=0$ and that $A / p$ is Cohen-Macaulay. Then $A / I$ is a Cohen-Macaulay ring of dimension $d$.

Lemma (3.3). $p \cap I=\left(a_{1}, a_{2}, \cdots, a_{r}\right)$.

Proof. Passing to the ring $A /\left(a_{1}, a_{2}, \cdots, a_{r}\right)$, we may assume that $r=0$. Let $x \in p \cap I$ and write $x=a_{1} y$ with $y \in A$. Then $a_{1} x=a_{1}^{2} y=0$, as $x \in I=(0): a_{1}$. Therefore $x=a_{1} y=0$, since $(0): a_{1}=(0): a_{1}^{2}$ by (2.5) (3) and we get that $p \cap I=(0)$.

Theorem (3.4). Assume that $A / p$ is Cohen-Macaulay. Then $G$ is a Cohen-Macaulay ring of $r(G)=r(A)$. 
Proof. As $s=d-r$, we see by (2.8) (1) that the local ring $G_{M}$ is Cohen-Macaulay whence $G$ is (cf. [11]). Let

$$
f_{i}=a_{i} \bmod p^{2}
$$

$(1 \leqq i \leqq r)$. Then it follows from (2.6) and [16, Theorem 1.1 and Proposition 2.6] that the forms $f_{1}, f_{2}, \cdots, f_{r}$ constitute a $G$-regular sequence and

$$
G /\left(f_{1}, f_{2}, \cdots, f_{r}\right) \cong G\left(p /\left(a_{1}, a_{2}, \cdots, a_{r}\right)\right)
$$

as $A$-algebras. Consequently, as $r(G)=r\left(G_{M}\right)$ (cf. [2, Theorem 3.1]), by (2.9) we may assume, passing to the ring $A /\left(a_{1}, a_{2}, \cdots, a_{r}\right)$, without loss of generality that $r=0$. Notice that in this situation all the $A$-modules $A / p, A / I$, and $I$ are Cohen-Macaulay and of dimension $d$ (cf. (3.2)).

Choose $t \in A$ so that $p=(0): t$. (Notice that $t \in I$ but $t \notin p$.) We put $a=a_{1}$.

Claim 1. $a+t$ is regular in $A$.

Proof. We put $b_{1}=a+t$. Then clearly $b_{1}$ is regular on $A / p$, as $b_{1} \notin p$. Let $x \in A$ and assume that $b_{1} x \in I$. Then $a \cdot b_{1} x=a^{2} x=0$, because $a t=0$ and $I=(0): a$. Hence $x \in I=(0): a^{2}$ and $b_{1}$ is regular on $A / I$. Since the ring $A$ is, by (3.3), embedded into the direct sum $A / p \oplus A / I$, we get that $b_{1}$ is regular also in $A$.

By this claim we find that the element $b_{1}=a+t$ can be extended to a system, say $b_{1}, b_{2}, \cdots, b_{d}$ of parameters for $A$. We put

$$
J=\left(b_{2}, b_{3}, \cdots, b_{d}\right) .
$$

Claim 2. $\operatorname{dim} A /(p+t I+J)=0$ and $r(G)=r(A /(p+t I+J))$.

Proof. We get $\operatorname{dim} A /(p+t I+J)=0$, as $p+t I+J \supset\left(b_{1}^{2}\right)+J$ (recall that $t \in I$ ).

Consider the second equality. Let $X$ be an indeterminate over $A$ and let $\varphi: A[X] \rightarrow R$ be the epimorphism of $A$-algebras such that $\varphi(X)$ $=a T$. Then as $I=(0): a=(0): a^{2}$, we get $\operatorname{Ker} \varphi=X \cdot I A[X]$ whence

$$
A[X] /(p A[X]+X \cdot I A[X]) \cong G
$$

as $A$-algebras (recall that $R / p R=G$ ). Consequently we have isomorphisms

$$
\text { (\#) } \begin{aligned}
A /(p+t I+J) & \cong A[X] /((X-t)+p A[X]+X \cdot I A[X]+J A[X]) \\
& \cong G /\left(X-t, b_{2}, b_{3}, \cdots, b_{d}\right) G
\end{aligned}
$$

of $A$-algebras. Thus, since $\operatorname{dim} A /(p+t I+J)=0$ as is proved above, 
we find that $G /\left(X-t, b_{2}, b_{3}, \cdots, b_{d}\right) G$ is an Artinian local ring whence $X-t, b_{2}, b_{3}, \cdots, b_{d}$ is a system of parameters for the Cohen-Macaulay local ring $G_{M}$. In particular

$$
r\left(G_{M}\right)=r\left(G /\left(X-t, b_{2}, b_{3}, \cdots, b_{a}\right) G\right)
$$

by (2.9). Hence the equality

$$
r(G)=r(A /(p+t I+J))
$$

follows from the isomorphisms (\#), as $r(G)=r\left(G_{M}\right)$ (cf. [2]).

Claim 3. There exist exact sequences

$$
\begin{array}{r}
0 \longrightarrow I /\left(b_{1}, b_{2}, \cdots, b_{d}\right) I \longrightarrow A /(p+t I+J) \stackrel{f}{\longrightarrow} \\
A /(p+I+J) \longrightarrow 0 \\
0 \longrightarrow I /\left(b_{1}, b_{2}, \cdots, b_{d}\right) I \longrightarrow A /\left(b_{1}, b_{2}, \cdots, b_{d}\right) \stackrel{g}{\longrightarrow} \\
A /(p+I+J) \longrightarrow 0
\end{array}
$$

of $A$-modules, where $f$ and $g$ are canonical epimorphisms.

Proof. (1) It suffices to show that

$$
(p+I+J) /(p+t I+J) \cong I /\left(b_{1}, b_{2}, \cdots, b_{d}\right) I
$$

as $A$-modules. Notice that $(p+I+J) /(p+t I+J) \cong I /(I \cap(p+J)+t I)$.

First of all, we shall prove that $I \cap(p+J)=J I$. Let $x \in I \cap(p+J)$ and write $x=a x_{1}+\sum_{i=1}^{d} b_{i} x_{i}$ with $x_{i} \in A(1 \leqq i \leqq d)$. Then as $a t=a x$ $=0$, we get

$$
a x_{1} \cdot(a+t)+\sum_{i=2}^{d} b_{i} \cdot\left(a x_{i}\right)=0 .
$$

Consequently $a x_{1} \in J$ because $b_{1}=a+t, b_{2}, \cdots, b_{d}$ is an $A$-regular sequence, whence we find that $x=a x_{1}+\sum_{i=2}^{d} b_{i} x_{i} \in J \cap I$. As $J \cap I=J I$ (recall that $b_{2}, b_{3}, \cdots, b_{d}$ is an $A / I$-regular sequence), this guarantees that

$$
I \cap(p+J)=J I
$$

as claimed.

Now recall that $t I=(a+t) I$ and we have

$$
(I \cap(p+J))+t I=J I+(a+t) I=\left(b_{1}, b_{2}, \cdots, b_{d}\right) I .
$$

Thus $(p+I+J) /(p+t I+J) \cong I /\left(b_{1}, b_{2}, \cdots, b_{d}\right) I$ as $A$-modules. 
(2) Because $p+I+J=I+\left(a+t, b_{2}, \cdots, b_{d}\right)$ and $b_{1}=a+t, b_{2}, \cdots, b_{d}$ is an $A / I$-regular sequence (cf. (3.2)), the required exact sequence (2) immediately follows from the short exact sequence

$$
0 \longrightarrow I \longrightarrow A \longrightarrow A / I \longrightarrow 0 \text {. }
$$

Now let us finish the proof of Theorem (3.4). We have only to prove that

$$
r(A /(p+t I+J))=r\left(A /\left(b_{1}, b_{2}, \cdots, b_{d}\right)\right),
$$

because $r(G)=r(A /(p+t I+J))$ by Claim 2 and $r(A)=r\left(A /\left(b_{1}, b_{2}, \cdots, b_{d}\right)\right)$ by (2.9). To do this, it suffices to show that

$$
f\left([(0): m]_{A /(p+t I+J)}\right)=(0)
$$

and

$$
g\left([(0): m]_{A /\left(b_{1}, b_{2}, \ldots, b_{d}\right)}\right)=(0)
$$

in the exact sequences obtained by Claim 3 (recall that

$$
r(A /(p+t I+J))=\operatorname{dim}_{A / m}[(0): m]_{A /(p+t I+J)}
$$

and

$$
r\left(A /\left(b_{1}, b_{2}, \cdots, b_{d}\right)\right)=\operatorname{dim}_{A / m}[(0): m]_{A /\left(b_{1}, b_{2}, \ldots, b d\right)}
$$

by definition).

Let $x \in A$ and assume that $m x \subset p+t I+J$. Then as $t x \in p+t I+J$, we may write $t(x-y) \in p+J$ with $y \in I$. Notice that $(a+t)(x-y) \in p$ $+J$, as $a \in p$. Then we get $x-y \in p+J$, because $b_{1}=a+t$ is regular on $A /(p+J)$. Thus $x \in p+I+J$, i.e.,

$$
f(x \bmod p+t I+J)=0 \quad \text { in } A /(p+I+J) .
$$

Let $x \in A$ and assume that $m x \subset\left(b_{1}, b_{2}, \cdots, b_{d}\right)$. Then $t x=(a+t) y+z$ for some $y \in A$ and $z \in J$. Because $t(x-y) \in p+J$, we get $x \in p+I+J$ as is mentioned above. Thus

$$
g\left(x \bmod \left(b_{1}, b_{2}, \cdots, b_{d}\right)\right)=0 \quad \text { in } A /(p+I+J),
$$

which completes the proof of Theorem (3.4).

Corollary (3.5). Assume that $A$ is a Gorenstein ring. Then the following three conditions are equivalent. 
(1) $G$ is a Gorenstein ring.

(2) $G$ is a Cohen-Macaulay ring.

(3) A/p is a Cohen-Macaulay ring.

Proof. (3) $\Rightarrow$ (1) See (3.4).

(1) $\Rightarrow(2)$ This is obvious.

$(2) \Rightarrow(3)$ By the same reason as in Proof of Theorem (3.4), we may assume that $r=0$. Then as $G$ is Cohen-Macaulay, we get $s \geqq d-1$ by (2.8) whence $A / I$ is, by (3.1), a Cohen-Macaulay ring of dimension $d$. Notice that (0): $I=p$, since $I$ is contained in $A / p$ as an ideal (cf. (3.3)). Then we find by [10, Proposition 3.1] that the ring $A / p$ is Cohen-Macaulay, because $A$ is Gorenstein by our standard assumption.

Proof of Theorem (1.1).

(2) $\Rightarrow$ (1) This follows from (3.4).

$(1) \Rightarrow(2)$ By (3.5) it is enough to show that $A$ is Gorenstein. We put

$$
R^{\prime}=R\left[T^{-1}\right]
$$

Then as is well-known,

$$
G \cong R^{\prime} / T^{-1} R^{\prime}
$$

as graded $A$-algebras. Let $N^{\prime}=m R^{\prime}+T^{-1} R^{\prime}+\left[R^{\prime}\right]_{+}$. Then $N^{\prime}$ is a unique graded maximal ideal in $R^{\prime}$ and the local ring $R_{N^{\prime}}^{\prime} / T^{-1} R_{N^{\prime}}^{\prime}=G_{M}$ is, by our assumption (1), Gorenstein. Therefore so is $R_{N^{\prime}}^{\prime}$, as $T^{-1}$ is $R^{\prime}$ regular. Hence we get by [2, Theorem 3.1] that $R^{\prime}$ is globally Gorenstein. Consequently the ring $A\left[T, T^{-1}\right]=R^{\prime}[T]$ is still Gorenstein and so we conclude that $A$ itself is a Gorenstein ring (notice that $A\left[T, T^{-1}\right]$ is a faithfully flat extension of $A$ ).

Example (3.6). Let $A=k[[X, Y, Z]]$ be a formal power series ring over a field $k$ and put

$$
p=\left(X^{3}-Y Z, Y^{2}-X Z, Z^{3}-X^{2} Y\right)
$$

in $A$. Then as $A / p$ is a Cohen-Macaulay ring (cf. (2.2)), we get by (3.5) that the ring $G(p)$ is Gorenstein.

Similarly let $A=k[[X, Y, Z, W]]$ be a formal power series ring and put

$$
p=\left(Z^{2}-Y W, X W-Y^{2} Z, Y^{3}-X Z\right)
$$


in $A$. Then $p$ is a prime ideal of $A$ with height 2 and is an almost complete intersection in $A$ (cf. Section 2). Moreover the ring $G(p)$ is Gorenstein, as $A / p$ is Cohen-Macaulay also in this case.

\section{$\S 4$. The Gorensteinness of Rees rings $R(p)$ and Proof of Theorem (1.2)}

We shall maintain the assumption and notation in the preface of Section 3.

First of all, we note the following

Lemma (4.1). The ring $R$ is not Cohen-Macaulay, if $r=0$.

Proof. The local ring $R_{N}$ is not Cohen-Macaulay, as depth $R_{\mathrm{v}} \leqq d$ by (2.8) (2) (recall that $\operatorname{dim} R_{N}=d+1$, cf., e.g., [17]).

Propositron (4.2). Suppose that $r \geqq 3$. Then the ring $R$ is not Gorenstein.

Proof. The ring $R_{p}=R\left(p A_{p}\right)$ cannot be Gorenstein, since $A_{p}$ is a regular local ring of $\operatorname{dim} A_{p}=r \geqq 3$ (cf., e.g., [5, Theorem (1.2)]).

Let $K$ be an $A$-module. Then $K$ is called the canonical module of $A$, if

$$
\hat{A} \underset{A}{\otimes} K \cong \operatorname{Hom}_{A}\left(H_{m}^{a}(A), E_{A}(A / m)\right)
$$

as $\hat{A}$-modules, where $\hat{A}$ (resp. $E_{A}(A / m)$ ) denotes the completion of $A$ (resp. the injective hull of $A / m$ ). The canonical module of $A$ is, if it exists, uniquely determined up to isomorphisms, which we denote by $K_{A}$. Various properties of canonical modules are discussed by [7]. Here let us summarize some of them, which we shall need later.

Proposition (4.3). (1) The ring $A$ possesses the canonical module if and only if $A$ is a homomorphic image of a Gorenstein ring.

(2) Suppose that $A$ possesses the canonical module $K_{A}$. Then

$$
v_{A}\left(K_{A}\right)=r(A) \text {. }
$$

(3) Let $K$ be an A-module. Then the following two conditions are equivalent:

(a) $K=K_{A}$.

(b) (i ) $K$ is a Cohen-Macaulay A-module of dimension d,

(ii) $[(0): K]_{A}=(0)$, and (iii) $\operatorname{dim}_{A / m}\left[(0): m_{H_{m}^{d}(K)}=1\right.$. 
(4) Suppose that $A$ contains the canonical module $K_{A}$ as a proper ideal. Then $A / K_{A}$ is a Gorenstein ring of dimension $d-1$.

Proof. (1) The if part is due to [7, 5.19]. See [13] for the only if part.

(2) See [7, Satz 6.10].

(3) (a) $\Rightarrow$ (b) See [7, Satz 6.1 and Lemma 6.6].

(b) $\Rightarrow$ (a) We may assume that $A$ is complete. First of all, recall that

$$
K \cong \operatorname{Hom}_{A}\left(\operatorname{Hom}_{A}\left(K, K_{A}\right), K_{A}\right)
$$

as $A$-modules since the $A$-module $K$ is Cohen-Macaulay and of dimension $d$ (cf. [7, Satz 6.1]). Notice that

$$
\operatorname{Hom}_{A}\left(K, K_{A}\right) \cong \operatorname{Hom}_{A}\left(H_{m}^{d}(K), E_{A}(A / m)\right)
$$

(cf. [7, Satz 5.2]) and we find, by the condition (iii), that the $A$-module $\operatorname{Hom}_{A}\left(K, K_{A}\right)$ is cyclic. Accordingly it follows from the condition (ii) that

$$
\operatorname{Hom}_{A}\left(K, K_{A}\right) \cong A
$$

because (0): $\operatorname{Hom}_{A}\left(K, K_{A}\right)=(0): K(\mathrm{cf} .(\#))$, whence we get by the isomorphism (\#) that $K=K_{A}$ as claimed.

(4) See [7, Korollar 6.13].

Theorem (4.4). Assume that $r=1$. Then the following two conditions are equivalent.

(1) $R$ is a Gorenstein ring.

(2) $K_{A}=\left(a_{1}\right): a_{2}$.

(Here $a_{1}, a_{2}$ are elements of $A$ obtained by (2.5).)

In order to prove Theorem (4.4) we need one more lemma. Let $A[X]$ be a polynomial ring over $A$ and let $P=m A[X]+X A[X]$, the unique graded maximal ideal of $A[X]$. We denote by $H_{P}^{i}(\cdot)$, for each $i$, the local cohomology functor relative to $P$. Given a graded $A[X]$-module $L$, we consider $H_{P}^{i}(L)$ to be a graded $A[X]$-module whose homogeneous component of degree $n$ shall be denoted by $\left[H_{P}^{i}(L)\right]_{n}(n \in \boldsymbol{Z})$.

Lemma (4.5). Let $E$ be a finitely generated A-module and $i$ an integer. Then

(1) $\left[H_{P}^{i+1}\left(A[X] \otimes_{A} E\right)\right]_{n}=(0)$ for all $n \geqq 0$.

(2) $\left[H_{P}^{i+1}\left(A[X] \otimes_{A} E\right)\right]_{n} \cong H_{m}^{i}(E)$ as A-modules for all $n<0$. 
Proof. For a given graded $A[X]$-module $L$, we denote by $L(-1)$ the graded $A[X]$-module whose underlying module coincides with that of $L$ and whose graduation is given by $[L(-1)]_{n}=L_{n-1}(n \in \boldsymbol{Z})$. With this notation, first of all, consider the exact sequence

$$
0 \longrightarrow(A[X] \underset{A}{\otimes} E)(-1) \stackrel{X}{\longrightarrow} A[X] \underset{A}{\otimes} E \longrightarrow E \longrightarrow 0
$$

of graded $A[X]$-modules. Apply functors $H_{P}^{i}(\cdot)$ to this sequence and we get a long exact sequence

$$
\begin{aligned}
\cdots \longrightarrow H_{m}^{i}(E) & \stackrel{\partial}{\longrightarrow} H_{P}^{i+1}(A[X] \underset{A}{\otimes} E)(-1) \\
& \stackrel{X}{\longrightarrow} H_{P}^{i+1}(A[X] \underset{A}{\otimes} E) \longrightarrow \cdots
\end{aligned}
$$

of local cohomology modules.

Now assume that $\left[H_{P}^{i+1}\left(A[X] \otimes_{A} E\right)\right]_{n} \neq(0)$ for some integer $n$. We choose such $n$ as large as possible. Then as

$$
X \cdot\left[H_{P}^{i+1}(A[X] \underset{A}{\otimes} E)(-1)\right]_{n+1}=(0),
$$

we get by the sequence $(\#)$ that

$$
(0) \neq\left[H_{P}^{i+1}(A[X] \underset{A}{\otimes} E)(-1)\right]_{n+1} \subset \partial\left(\left[H_{m}^{i}(E)\right]_{n+1}\right),
$$

whence $\left[H_{m}^{i}(E)\right]_{n+1} \neq(0)$. Thereby we get $n+1=0$, because $\left[H_{m}^{i}(E)\right]_{q}=$ (0) for all $q \neq 0$. Thus

$$
\left[H_{P}^{i+1}(A[X] \underset{A}{\otimes} E)\right]_{n}=(0)
$$

for all integers $n \geqq 0$ and $i$.

Let us prove the assertion (2). Let $n<0$ and $i$ be integers. Consider the homogeneous component of degree $n$ (resp. degree 0 ) in the sequence (\#) and, because $H_{m}^{j}(E)=\left[H_{m}^{j}(E)\right]_{0}$ (resp. $\left[H_{P}^{j}\left(A[X] \otimes_{A} E\right)\right]_{0}=(0)$ by the assertion (1)) for all $j$, we get an isomorphism

$$
\begin{aligned}
& {\left[H_{P}^{i+1}\left(A[X] \otimes_{A} E\right)\right]_{n-1} \cong\left[H_{P}^{i+1}(A[X] \underset{A}{\otimes} E)\right]_{n}} \\
& \left(\text { resp. } H_{m}^{i}(E) \cong\left[H_{P}^{i+1}(A[X] \underset{A}{\otimes} E)\right]_{-1}\right)
\end{aligned}
$$

of $A$-modules. Hence

$$
\left[H_{P}^{i+1}(A[X] \underset{A}{\otimes} E)\right]_{n} \cong H_{m}^{i}(E)
$$

for all integers $n<0$ and $i$. 
Proof of Theorem (4.4).

Let $A[X, Y]$ be a polynomial ring over $A$ and let $\varphi: A[X, Y] \rightarrow R$ be the epimorphism of graded $A$-algebras defined by $\varphi(X)=a_{2} T$ and $\varphi(Y)$ $=a_{1} T$. Then by virtue of (2.7) we find that

$$
\operatorname{Ker} \varphi=\left(b_{2} X+b_{1} Y \mid b_{1}, b_{2} \in A \text { such that } a_{1} b_{1}+a_{2} b_{2}=0\right)
$$

in $A[X, Y]$. Hence

$$
A[X] / X \cdot I A[X] \cong R /\left(a_{1} T\right)
$$

as graded $A$-algebras, where $I=\left(a_{1}\right): a_{2}$.

(1) $\Rightarrow(2)$ First of all, notice that $s \geqq d-2$ by (2.8) (2), as $R$ is Cohen-Macaulay and as $r=1$. Then we get by (3.1) that $A / I$ is a CohenMacaulay ring of dimension $d-1$. Hence the $A$-module $I$ is CohenMacaulay and of dimension $d$.

Now apply functors $H_{P}^{i}(\cdot)$ (here $P=m A[X]+X A[X]$ ) to the exact sequence

$$
0 \longrightarrow(I A[X])(-1) \longrightarrow A[X] \longrightarrow A[X] / X \cdot I A[X] \longrightarrow 0
$$

of graded $A[X]$-modules. Then we obtain an exact sequence

(b) $0 \longrightarrow H_{P}^{d}(A[X] / X \cdot I A[X]) \longrightarrow H_{P}^{d+1}(I A[X])(-1) \longrightarrow H_{P}^{d+1}(A[X])$

of local cohomology modules. Recall that

$$
\left[H_{P}^{d+1}(A[X])\right]_{0}=(0) \quad \text { and } \quad\left[H_{P}^{d+1}(I A[X])\right]_{n}=(0) \quad(n \geqq 0)
$$

by (4.5) (1). Then we get, by the above sequence (b) and (4.5) (2), that

$$
\begin{aligned}
& {\left[H_{P}^{d}(A[X] / X \cdot I A[X])\right]_{n}=(0) \quad(n>0) \quad \text { and }} \\
& {\left[H_{P}^{d}(A[X] / X \cdot I A[X])\right]_{0}=H_{m}^{d}(I) .}
\end{aligned}
$$

On the other hand, recalling that $R /\left(a_{1} T\right)$ is a Gorenstein ring as $R$ is Gorenstein by our assumption (1) and as $a_{1} T$ is a regular element of $R$, we find by the isomorphism (a) that the ring $A[X] / X \cdot I A[X]$ is also Gorenstein. Hence

$$
\operatorname{dim}_{A[X] / P}[(0): P]_{H_{P}^{d}(A[X] / X \cdot I A[X])}=1
$$

and consequently, by the assertion (c), we get that

$$
\operatorname{dim}_{A / m}[(0): m]_{H_{m}^{d}(I)}=1 .
$$

Since $I$ is a Cohen-Macaulay $A$-module of dimension $d$ and since 
$[(0): I]_{A}=(0)$ (recall that $I$ contains a non-zerodivisor $a_{1}$ of $A$ ), we conclude by (4.3) (3) that $I=K_{A}$ as required.

$(2) \Rightarrow(1)$ We have by the assumption (2) that

$$
K_{A[X]_{P}}=X \cdot I A[X]_{P},
$$

since $I A[X] \cong X \cdot I A[X]$ as $A[X]$-modules and $K_{A[X]_{P}}=A[X]_{P} \otimes_{A} K_{A}$ (cf. [7, Korollar 5.21]). Hence, by (4.3) (4), the local ring $A[X]_{P} / X \cdot I A[X]_{P}$ is Gorenstein and thereby we get, by the isomorphism (a), that so is the ring $R_{N} /\left(a_{1} T\right) R_{N}$. Because $a_{1} T$ is $R$-regular, $R_{N}$ is Gorenstein and thus we have by [2, Theorem 3.1] that $R$ is globally a Gorenstein ring. This completes the proof of Theorem (4.4).

Corollary (4.6). Assume that $r=1$ and that $A$ is Gorenstein. Then $R$ is not a Gorenstein ring.

Proof. Suppose that $R$ is Gorenstein. Then we have, by (4.3) (2) and (4.4), that

$$
v_{A}\left(\left(a_{1}\right): a_{2}\right)=1
$$

Let $I=\left(a_{1}\right): a_{2}=(b)$ with $b \in A$.

Claim. $b \notin p$.

Proof. If $b \in p$, then we get $I=\left(a_{1}\right)$ as $p \cap I=\left(a_{1}\right)$ by (3.3). Hence $a_{1}, a_{2}$ is an $A$-regular sequence and so we have $r=\operatorname{dim} A_{p}=2$ - this is impossible.

We write $a_{1}=b x$ and $a_{2} b=a_{1} y$ with $x, y \in A$. Then $x \in p=\left(a_{1}, a_{2}\right)$, since $b \notin p$ by the above claim. Express $x=a_{1} z+a_{2} w$ with $z, w \in A$. Then as $a_{1}$ is $A$-regular and as

$$
\begin{aligned}
a_{1} & =b x \\
& =a_{1} \cdot b z+a_{2} b \cdot w \\
& =a_{1} \cdot b z+a_{1} \cdot y w,
\end{aligned}
$$

we get $1=b z+y w$ whence $y$ is a unit of $A$. Hence $a_{1} \in\left(a_{2}\right)$, as $a_{2} b=$ $a_{1} y$ by our choice and therefore $p=\left(a_{2}\right)$ - this contradicts our standard assumption that $p$ is minimally generated by 2 elements. Thus $R$ is not a Gorenstein ring.

ExAmple (4.7). Let $k[[s, t, u]]$ be a formal power series ring over a field $k$. We put 


$$
A=k\left[\left[s^{2}, t, s^{3}, s t, s^{2} u, t u\right]\right]
$$

in $k[[s, t, u]]$ and

$$
p=\left(s^{2} u, t u\right) A
$$

Then

(1) $A$ is a Cohen-Macaulay local domain of $\operatorname{dim} A=3$;

(2) $p$ is a prime ideal of height 1 and is an almost complete intersection in $A$;

(3) the ring $R(p)$ is Gorenstein;

(4) the ring $G(p)$ is Cohen-Macaulay but not Gorenstein.

Proof. (1) As $B=k\left[\left[s^{2}, t, s^{3}, s t\right]\right]$ is a Buchsbaum local domain of dimension 2 and as $s^{2}, t$ is a system of parameters for $B$, we know by [15] that $A$ is a Cohen-Macaulay ring of dimension 3.

(2) As $B=A / p$, the ideal $p$ is prime and $\operatorname{dim} A_{p}=1$. Clearly $p$ is an almost complete intersection in $A$ (recall that $p A_{p}=\left(s^{2} u\right) A_{p}$ ).

(3) Notice that the elements $a_{1}=s^{2} u$ and $a_{2}=t u$ fulfill the conditions (1), (2), and (3) in Lemma (2.5). Let $I=\left(s^{2} u\right): t u$ in $A$. Then as $\operatorname{depth} A / p=1$, we get by (3.1) that $A / I$ is a Cohen-Macaulay ring of dimension 2. Hence the $A$-module $I$ is Cohen-Macaulay. Therefore in order to prove that the ring $R(p)$ is Gorenstein, it suffices, by (4.3) (3) and (4.4), to show that

$$
\operatorname{dim}_{A / m}[(0): m]_{H_{m}^{s}(I)}=1 .
$$

Let $r_{A}(I)=\operatorname{dim}_{A / m} \operatorname{Ext}_{A}^{3}(A / m, I)$. Then we get, by [7, Satz 5.2 and Satz 6.10], that

$$
\begin{aligned}
\operatorname{dim}_{A / m}[(0): m]_{H_{m}^{3}(I)} & =v_{A}\left(\operatorname{Hom}_{A}\left(H_{m}^{3}(I), E_{A}(A / m)\right)\right) \\
& =v_{A}\left(\operatorname{Hom}_{A}\left(I, K_{A}\right)\right) \\
& =r_{A}(I) .
\end{aligned}
$$

Hence we have only to show that $r_{A}(I)=1$. Notice that $s^{2}, t-s^{2} u, t u$ is a system of parameters for $A$ and that

$$
I=\left(s^{2} u, s^{2}, s^{3}\right) A \text {. }
$$

Then as

$$
r_{A}(I)=\operatorname{dim}_{A / m}[(0): m]_{I /\left(s^{2}, t-s^{2} u, t u\right) I}
$$

by (2.9), it is now routine to check that $r_{A}(I)=1$, which we leave to the reader. 
(4) The assertion that $G(p)$ is not Gorenstein follows from (1.1), because $A / p(=B)$ is not a Cohen-Macaulay ring in this example (see also (4.6)). Of course $G(p)$ is Cohen-Macaulay by (2.8) (1).

The rest of this section is devoted to a proof of Theorem (1.2). Notice that the assertion (1) in Theorem (1.2) is already given by (4.2). To prove the assertion (2) we need a few preliminaries.

In what follows, we assume that $r=2$ and put

$$
\bar{A}=A / a_{1} A, \quad \bar{p}=p / a_{1} A, \quad \text { and } \quad \bar{R}=R(\bar{p}) .
$$

Let $\varphi: R \rightarrow \bar{R}$ be the epimorphism of Rees rings induced by the canonical map $A \rightarrow \bar{A}$. Let $A[X, Y, Z]$ be a polynomial ring over $A$ and let $\psi: A[X, Y, Z] \rightarrow R$ denote the homomorphism of $A$-algebras defined by

$$
\psi(X)=a_{1} T, \quad \psi(Y)=a_{2} T, \text { and } \psi(Z)=a_{3} T .
$$

Then we have the following

Lemma (4.8). (1) $\operatorname{Ker} \varphi=\left(a_{1}, a_{1} T\right)$.

(2) $\operatorname{Ker} \psi=\left(b_{1} X+b_{2} Y+b_{3} Z \mid b_{1}, b_{2}, b_{3} \in A\right.$ such that $a_{1} b_{1}+a_{2} b_{2}+a_{3} b_{3}$ $=0)$.

Proof. (1) This follows from the equality $a_{1} A \cap p^{n}=a_{1} p^{n-1}(n>0)$, cf. (2.6).

(2) See (2.7).

Corollary (4.9). There exists an exact sequence

$$
0 \longrightarrow G(-1) \longrightarrow R / a_{1} R \longrightarrow \bar{R} \longrightarrow 0
$$

of graded $R$-modules.

Proof. Notice that

$$
\begin{aligned}
\left(a_{1}, a_{1} T\right) / a_{1} R & \cong\left(a_{1} T\right) /\left(a_{1} T\right) \cap a_{1} R \\
& =\left(a_{1} T\right) / a_{1} T \cdot p R \\
& \cong(R / p R)(-1)
\end{aligned}
$$

as graded $R$-modules. Then since $G=R / p R$, we get by (4.8) (1) the required exact sequence at once.

We put

$$
Q=\left(a_{1} T, a_{2} T-a_{1}, a_{3} T-a_{2}\right) R
$$

and 


$$
\begin{array}{r}
q=\left(a_{1} b_{2}+a_{2} b_{3} \mid b_{2}, b_{3} \in A \text { such that } a_{1} b_{1}+a_{2} b_{2}+a_{3} b_{3}=0\right. \\
\text { for some } \left.b_{1} \in A\right) .
\end{array}
$$

LEMMA (4.10). (1) $q \supset\left(a_{1}^{2}, a_{2}^{2}-a_{1} a_{3}, a_{1} a_{2}\right)$.

(2) $A / q \cong R / Q$ as A-algebras.

(3) $\operatorname{dim} A / q=d-2$.

Proof. (1) Notice that $a_{1}\left(-a_{2}\right)+a_{2} a_{1}=0, a_{2} a_{3}+a_{3}\left(-a_{2}\right)=0$, and $a_{1}\left(-a_{3}\right)+a_{3} a_{1}=0$.

(2) We have an isomorphism

$$
A[X, Y, Z] /\left(\left(X, Y-a_{1}, Z-a_{2}\right)+\operatorname{Ker} \psi\right) \cong R / Q
$$

of $A$-algebras. Notice that, by (4.8) (2), $A / q$ is isomorphic to the ring on the left hand side.

(3) We get $\operatorname{dim} A / q \leqq d-2$ by the assertion (1), since $a_{1}^{2}, a_{2}^{2}-a_{1} a_{3}$ is an $A$-regular sequence. Recall that $\operatorname{dim} R_{N}=d+1$ and that the ideal $Q$ is generated by 3 elements in $N$. Then we have

$$
\operatorname{dim} R / Q \geqq \operatorname{dim} R_{N} / Q R_{N} \geqq d-2,
$$

whence $\operatorname{dim} A / q=\operatorname{dim} R / Q=d-2$ (recall that $A / q \cong R / Q$ by the assertion (2)).

Now we are ready to prove Theorem (1.2).

Proof of Theorem (1.2) (2).

Assume that $R$ is Gorenstein. Then we have $s \geqq d-3$ by (2.8) (2). Hence by (2.8) (2) (resp. (2.8) (1)), the ring $\bar{R}$ (resp. $G$ ) is a CohenMacaulay ring of dimension $d$. Therefore applying functors $H_{N}^{i}(\cdot)$ to the exact sequence obtained by (4.9), we get a short exact sequence

$$
0 \longrightarrow\left[H_{M}^{d}(G)\right](-1) \longrightarrow H_{N}^{d}\left(R / a_{1} R\right) \longrightarrow H_{N}^{d}(\bar{R}) \longrightarrow 0
$$

of local cohomology modules. Recall that $\operatorname{dim}_{R / N}[(0): N]_{H_{N}^{d}\left(R / a_{1} R\right)}=1$, as the ring $R / a_{1} R$ is Gorenstein. Then we have by the sequence (\#) that

$$
\operatorname{dim}_{G / M}[(0): M]_{H_{M}^{d}(G)}=1,
$$

whence $G$ is a Gorenstein ring.

Conversely suppose that the ring $G$ is Gorenstein. Then by (1.1) $A$ is Gorenstein and $A / p$ is Cohen-Macaulay. Hence $R$ is, by (2.8), CohenMacaulay. Since $a_{1} T, a_{2} T-a_{1}, a_{3} T-a_{2}$ is a subsystem of parameters for the local ring $R_{N}$ and since $A / q \cong R /\left(a_{1} T, a_{2} T-a_{1}, a_{3} T-a_{2}\right)$ (cf. (4.10), 
(2) and (3)), in order to prove that the ring $R$ is Gorenstein it suffices to show that so is the ring $A / q$.

Let $J=\left(a_{1}^{2}, a_{2}^{2}-a_{1} a_{3}\right) A$. Then as $A$ is Gorenstein and as $a_{1}^{2}, a_{2}^{2}-a_{1} a_{3}$ is an $A$-regular sequence, the ring $A / J$ is again a Gorenstein ring of dimension $d-2$. Recall that $q \supset J$ and that $A / q(=R / Q)$ is a CohenMacaulay ring of dimension $d-2$. Then we find by [10, Proposition (3.1) (c)] that

$$
r(A / q)=v_{A}((J: q) / J) .
$$

Lemma (4.11). $J: q=J+\left(a_{2}\right)$.

Proof. Let $b_{1}, b_{2}, b_{3}$ be elements of $A$ such that $a_{1} b_{1}+a_{2} b_{2}+a_{3} b_{3}=0$. Then as $a_{2}^{2} \equiv a_{1} a_{3} \bmod J$, we find that

$$
\begin{aligned}
a_{2} \cdot\left(a_{1} b_{2}+a_{2} b_{3}\right) & \equiv a_{1} \cdot\left(a_{2} b_{2}+a_{3} b_{3}\right) \\
& \equiv a_{1} \cdot\left(-a_{1} b_{1}\right) \\
& \equiv 0
\end{aligned}
$$

$\bmod J$. Hence $a_{2} q \subset J$.

Conversely let $x \in J: q$. First of all, we shall show that $x \in\left(a_{1}, a_{2}\right)$. In fact, as $a_{1} a_{2} \in q$ (cf. (4.10) (1)), we may write

$$
a_{1} a_{2} x=a_{1}^{2} y+\left(a_{2}^{2}-a_{1} a_{3}\right) z
$$

with $y, z \in A$. Hence $z \in\left(a_{1}\right)$, as $a_{1}, a_{2}^{2}-a_{1} a_{3}$ is an $A$-regular sequence. Let $z=a_{1} v$ with $v \in A$. Then we get, as $a_{2} x=a_{1} y+\left(a_{2}^{2}-a_{1} a_{3}\right) v$, that $a_{2}\left(x-a_{2} v\right) \in\left(a_{1}\right)$. Hence $x \in\left(a_{1}, a_{2}\right)$ as required.

Now let us write $x=a_{1} f+a_{2} g$ with $f, g \in A$. Then $a_{1} f \in J: q$, because $a_{2} \in J: q$ as is proved earlier. Let $t$ be an element of $A$ such that $p=$ $\left(a_{1}, a_{2}\right): t$. We express $a_{1} b_{1}+a_{2} b_{2}+a_{3} t=0$ with $b_{1}, b_{2} \in A$. Then $a_{1} b_{2}+$ $a_{2} t \in q$ by definition, whence $a_{1} f \cdot\left(a_{1} b_{2}+a_{2} t\right) \in J$. We write

$$
a_{1} f \cdot\left(a_{1} b_{2}+a_{2} t\right)=a_{1}^{2} h+\left(a_{2}^{2}-a_{1} a_{3}\right) k
$$

with $h, k \in A$. Notice that $k \in\left(a_{1}\right)$, because $a_{1}, a_{2}^{2}-a_{1} a_{3}$ is an $A$-regular sequence. Let $k=a_{1} k^{\prime}$ with $k^{\prime} \in A$. Then

$$
f \cdot\left(a_{1} b_{2}+a_{2} t\right)=a_{1} h+\left(a_{2}^{2}-a_{1} a_{3}\right) k^{\prime}
$$

clearly and so we find that

$$
a_{2}\left(f t-a_{2} k^{\prime}\right) \in\left(a_{1}\right) .
$$

Therefore $f t \in\left(a_{1}, a_{2}\right)$ as $a_{1}, a_{2}$ is an $A$-regular sequence, whence $f \in p=$ $\left(a_{1}, a_{2}\right): t$. Because $p=\left(a_{1}, a_{2}, a_{3}\right)$, we get that

$$
x=a_{1} f+a_{2} g \in\left(a_{1}^{2}, a_{2}, a_{1} a_{3}\right)=J+\left(a_{2}\right) .
$$


Thus $J: q=J+\left(a_{2}\right)$ as claimed.

By this lemma (4.11) we find that $r(A / q)=1$ and hence the ring $R$ is Gorenstein. This completes the proof of Theorem (1.2) (2).

\section{REFERENCES}

[1] D. F. Anderson, Subrings of $k[X, Y]$ generated by monomials, Canad. J. Math., 30 (1978), 215-224.

[2] Y. Aoyama and S. Goto, On the type of graded Cohen-Macaulay rings, J. Math. Kyoto Univ., 15 (1975), 19-23.

[ 3 ] M. Artin, On isolated rational singularities of surfaces, Amer. J. Math., 88 (1966), 129-137.

[4] M. Brodman, On generic complete intersection ideals, in preprint.

[5] S. Goto and Y. Shimoda, On the Rees algebras of Cohen-Macaulay local rings, Lecture Notes in Pure and Appl. Math., 68 (1982), 201-231, Marcel Dekker, Inc.

[6] J. Herzog, Generators and relations of abelian semigroups and semigrouprings, Manuscripta Math., 3 (1970), 175-193.

[ 7 ] J. Herzog and E. Kunz, Der kanonische Modul eines Cohen-Macaulay-Rings, Lecture Notes in Math., 238 (1971), Springer-Verlag.

[ 8 ] J. Herzog, A. Simis and W. Vasconcelos, Approximation complexes of blowing-up rings, J. Algebra, 74 (1982), 466-493.

[9] C. Huneke, Symbolic powers of primes and special graded algebras, to appear in Comm. Algebra.

[10] E. Kunz, Almost complete intersections are not Gorenstein rings, J. Algebra, 28 (1974), 111-115.

[11] J. Matijevic and P. Roberts, A conjecture of Nagata on graded Cohen-Macaulay rings, J. Math. Kyoto Univ., 14 (1974), 125-128.

[12] M. P. Murthy, A note on the 'Primbasissatz', Arch. Math., 12 (1961), 425-428.

[13] I. Reiten, The converse to a theorem of Sharp on Gorenstein modules, Proc. Amer. Math. Soc., 32 (1972), 417-420.

[14] J. Sally, Cohen-Macaulay local rings of maximal embedding dimension, J. Algebra, 56 (1979), 168-183.

[15] Y. Shimoda, A note on Rees algebras of two dimensional local domains, J. Math. Kyoto Univ., 19 (1979), 327-333.

[16] P. Valabrega and G. Valla, Form rings and regular sequences, Nagoya Math. J., 72 (1978), 93-101.

[17] G. Valla, Certain graded algebras are always Cohen-Macaulay, J. Algebra, 42 (1976), 537-548.

Shiro Goto:

Department of Mathematics

Nihon University

3-25-40 Sakurajosui

Setagaya-ku, Tokyo 156

Yasuhiro SHIMODA:

Jôhoku Gakuen High School

2-28-1 Tôshin-cho

Itabashi-ku, Tokyo 174 